\title{
Neuropharmacological therapy of polycythemia vera: roles of circulating catechola- mines and serotonin
}

$\mathrm{P}$ olycythemia Vera (PV) is a myeloproliferative disorder of unknown cause. Erythrocytosis is usually the dominant presenting feature. Earlier studies of the pathogenesis focused on levels of erythropoietin, the erythropoietin receptors, and the oxyhemoglobin dissociation curve, but no significant differences were found between these patients and normal control subjects. More recent studies confirm that the defect is, at the stem cell level, consistent with the natural history of PV when treated by phlebotomy alone, leading to the development of myelofibrosis, acute myeloid leukemia (AML) and myelodysplasia. Moreover, the incidence of AML in PV patients is exacerbated by treatment with alkylating agents, phosphorus-32 and hydroxyurea (1).

Concomitant erythromelalgia of the hand palm was confirmed by histopathological findings of arteriolar thrombotic lesions in the reticular dermis in the two PV patients reported in this trial. The increased hematocrit in these patients contributed to progression of the microvascular syndrome of thrombocythemia into major occlusive ischemic events of the extremities. Standard therapy with oral anticoagulants and reduction of the hematocrit to normal by blood-letting did not affect the platelet-mediated microvascular erythromelalgic, ischemic symptoms because thrombocythemia vera persisted (1).

\section{Patient A}

N.P.M. is currently a 52-year-old white non-smoker and non-depressed woman who was diagnosed with PV in June, 1984. Pulmonary, cardiac, gastrointestinal and renal diseases were excluded. She was treated with hydroxyurea and phlebotomy until April 2002, when she became unresponsive to these therapies. She presented painful red, blue and black toe and finger syndrome. Haematological investigation showed white blood cell count $(\mathrm{WBC})=17.1 \times 10^{3}$, haematocrit $(\mathrm{H})=62.6 \%$ and platelet count $(\mathrm{P})=713 \times 10^{3}$. Splenomegaly and pulmonary emphysema were detected. The investigation of circulating neurotransmitters showed raised levels of noradrenaline (NA) and platelet serotonin (p-5HT), plus lowered levels of plasma tryptophane (trp). Adrenaline (Ad), dopamine (DA) and free-serotonin in the plas-

\footnotetext{
Correspondence to:

Fuad Lechin, MD, PhD

Apartado 80.983

Caracas 1080-A

Venezuela

E-mail: Oflechin@telcel.net.ve

Tel.: +58 212961 1048, Fax: +58 2129610172

Received August 17, 2004

Accepted after resubmission October 5, 2004
}

Thromb Haemost 2005; 93: I75-7 ma (f-5HT) showed normal values. The NA/Ad ratio was greater than normal (7.3), (normal $=3$ ). This NA/Ad ratio increased at one-minute orthostasis and five-minute exercise challenges. This sympathetic profile is consistent with predominance of neural vs adrenal activity (2).

Immunological investigation showed increased CD4/CD8 ratio (5.3), increased IgG in the plasma, positive antinuclear antibodies $(++)$ and increased natural killer (NK) cell cytotoxicity against K-562 target cells. Summarizing all the above: central nervous system (CNS)-NA over CNS-5HT predominance and TH-1 (T-helper inducer cells $=$ tissue immunity) over TH-2 (T-cytotoxic/suppressor cells $=$ humoral immunity) predominance were detected (3). We prescribed a neuropharmacological therapy to enhance CNS serotonergic activity. Thus, oral serotonin precursor (5-OH-tryptophane; $25 \mathrm{mg}$ three times daily) plus paroxetine $20 \mathrm{mg}$ daily (a 5HT-uptake inhibitor) were prescribed (4). This treatment was started in May 2002 and continues to the present day. Normalization of clinical, haematological, immunological and neurochemical parameters (including NA/Ad ratio) was obtained six weeks after starting neuropharmacological therapy. Significant and progressive reductions of p-5HT were also registered. To date, all levels remain normal (September 2004). Treatment was interrupted for two weeks after each 12 -week treatment period. No relapses have been registered to date.

\section{Patient B}

F.R. is currently a 62-year-old white non-smoker and non-depressed man, who was diagnosed with PV in July, 2001. He was treated with hydroxyurea and phlebotomy until May, 2002 when he became unresponsive to those therapies and was referred to our institute. Haematological investigation performed at this time showed $\mathrm{WBC}=18.3 \times 10^{3}, \mathrm{H}=64.1 \%$ and $\mathrm{P}=755 \times 10^{3}$. Splenomegaly and severe dermatological signs were evident. Coronary accidents plus pulmonary symptoms were frequently observed and treated in emergency hospitals. Painful red and blue toe and finger syndrome was also present. Standard therapy with oral anticoagulants and reduction of the hematocrit to normal by blood-letting did not affect the platelet-mediated microvascular erythromelalgic, ischemic symptoms.

The investigation of circulating neurotransmitters showed a profile similar to that of patient A. Raised NA/Ad ratio, high p-5HT and low trp values were found at the time he was tested in our institute (May, 2002). The NA/Ad ratio showed additional increase throughout the supine-resting plus one-minute orthostasis and five-minute exercise test. This CNS-NA predominance is reflected at peripheral level by a greater neural sympathetic than adrenal sympathetic activity. On the other hand, the low trp circulating values reflect a poor CNS serotonergic activity. Sig- 
Table I: Ratios of haematocrit (Ht), platelet count, white blood cell (WBC) count, platelet serotonin ( $p$-5HT), noradrenaline (NA) I adrenaline (Ad) before and after neuropharmacological treatment aimed at increasing serotonergic activity at the central nervous system in two polycythemia vera patients.

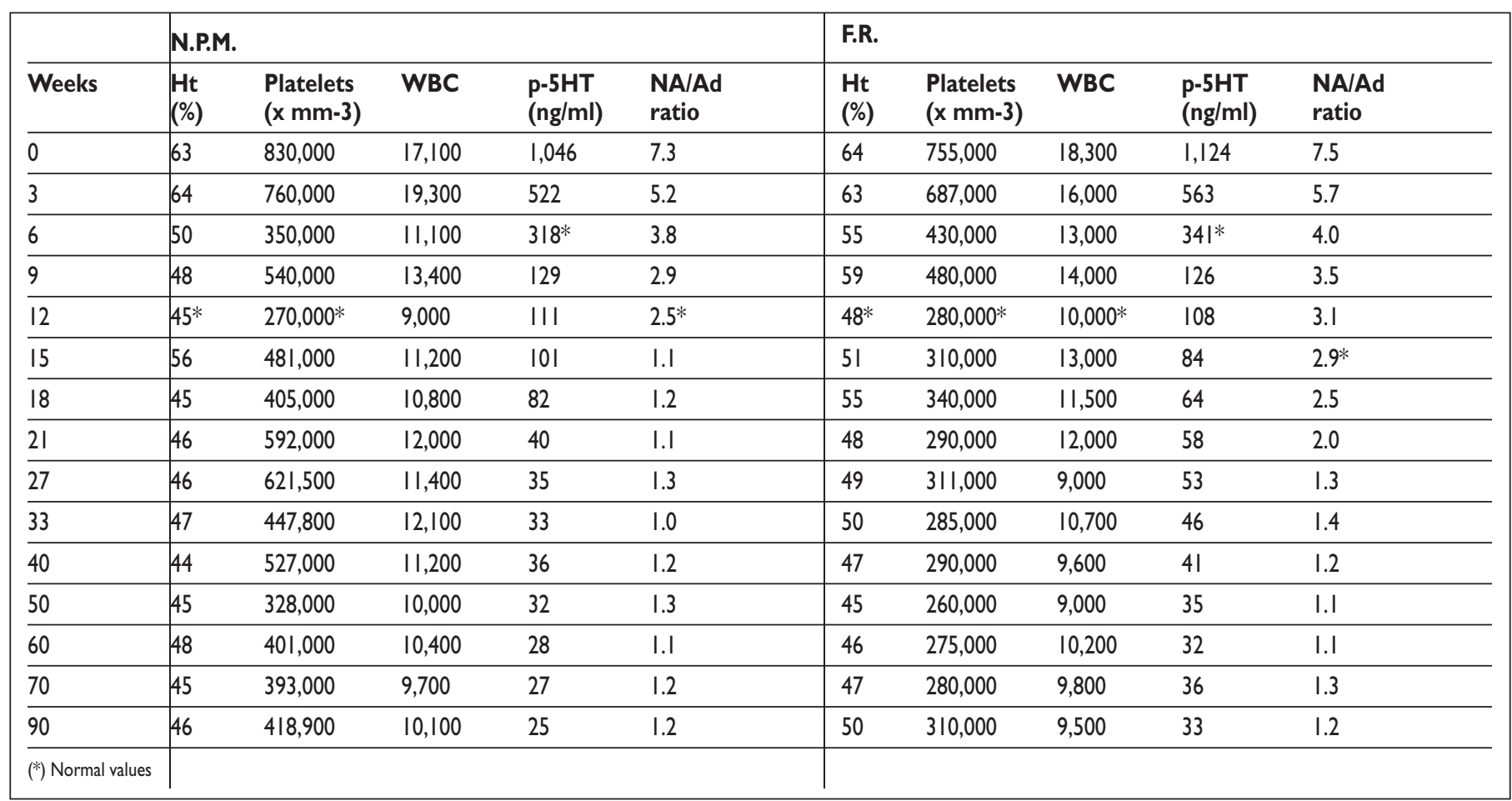

nificant reductions of the NA/Ad ratio and p-5HT values were progressively observed.

Immunological investigation also showed a similar profile to that registered in patient $\mathrm{A}$, consistent with the statement of $\mathrm{TH}-1$ over TH-2 autoimmune predominance. A similar neuropharmacological therapy to case A was started in this patient in August 2002 , continuing to the present. All parameters (clinical, haematological, neurochemical and immunological) were normalized six weeks after the neuropharmacological therapy began and remain normal to date. (September 2004). No side effects have been observed in these two PV patients during the neuropharmacological treatment.

\section{Discussion}

The above findings are consistent with the fact that the two PV cases reported here were associated with a noradrenergic over serotonergic predominance at CNS level. In addition, this CNSNA over 5-HT predominance was paralleled by a TH-1 over TH-2 immune profile (3). Finally, normalization of the neurochemical CNS disorder was paralleled by restoration of both the normal ANS profile and the immunological peripheral balance.

It is important to emphasize the role played by $\mathrm{p}-5 \mathrm{HT}$ as an important thrombogenic factor. We have demonstrated that the platelet content of serotonin rather than the platelet count is the most important factor involved in haemostasis $(2,5,6)$. We observed that disappearance of thrombotic episodes (Patient B) occurred three weeks after the start of neuropharmacological therapy, at which time p-5HT but not platelet count, dropped. This finding is the mirror image of that observed in our thrombocytopenic purpura patients, in whom the bleeding stoppage was paralleled by the $\mathrm{p}-5 \mathrm{HT}$ increase rather than the platelet number increase $(2,7)$. We discussed this phenomenon recently in several papers and assigned a fundamental role to $\mathrm{p}-5 \mathrm{HT}$ in all types of thrombotic events, which can be eliminated by lowering platelet serotonin content with drugs that interfere with serotonin uptake by both platelets and serotonergic neurons (8-11).

Finally, reversion of the TH-1 immunological profile seen in our PV patients, following reversion of the CNS-NA over 5-HT predominance, suggests that this immunological disorder plays an important etiopathogenic role in triggering relapse periods.

It is known that the spleen bone marrow and all haematopoietic organs receive neural sympathetic innervation. Sympathetic nerves arise from the lumbar spinal sympathetic neurons that send pre-ganglionic axons to sympathetic ganglia. Such preganglionic branches contact postganglionic sympathetic neurons (located at this level), which innervate the above-mentioned haematopoietic organs. In turn, spinal sympathetic neurons receive axons from the LC-A6 NA pontine nucleus, which is the main center responsible for neural sympathetic activity.

The CNS serotonergic system is composed of several 5HT nuclei. Dorsal Raphe (DR) and Median Raphe (MR) serotonin pontine nuclei send axons which bridle the LC-NA nucleus. Thus, any deficit of the CNS serotonergic activity will redound in CNS-NA predominance.

The peripheral serotonergic system depends on enterochromaffin cells. Parasympathetic drive excites these cells which release 5HT. Most serotonin is released to the intestinal lumen 
where it excites gastrointestinal motility. Some fraction of 5HT is released to portal circulation. Serotonin which escapes from liver and lung uptake is trapped by platelets $(=\mathrm{p}-5 \mathrm{HT})$. There are drugs which facilitate platelet uptake (such as tianeptine), other drugs like paroxetine interfere with serotonin uptake by platelets. We found that tianeptine is able to suddenly stop bleeding in patients affected by thrombocytopenic purpura. Conversely, we have found that drugs like paroxetine, interfere with thrombogenesis and thrombostasis.
Fuad Lechin, Bertha van der Dijs, Beatriz Orozco, Simon Rodriguez, Scarlet Baez

Department of Physiological Sciences, Sections of Neurochemical, Neurophysiology, Neuroimmunology and Neuropharmacology, Instituto de Medicina Experimental, Universidad Central de Venezuela, Caracas, Venezuela

\section{References}

1. Hocking WG. Primary and secondary erythrocytosis. In: Mazza JJ (Ed) Manual of clinical hematology. New York: Lippincott Williams \& Wilkins 2002; 73-89. 2. Lechin F, van der Dijs B, Orozco B, et al. Neuropharmacological treatment of refractory idiopathic thrombocytopenic purpura: Roles of circulating catecholamines and serotonin. Thromb Haemost 2004; 91 : 1254-6.

3. Lechin F, van der Dijs B, Lechin ME. Neuroautonomic, neuroendocrine, neuroimmune interactions. In: Lechin F, van der Dijs B, Lechin ME (eds). Neurocircuitry and Neuroautonomic Disorders: Reviews and Strategies of Therapy. Basel: Karger 2002; 57-9

4. Lechin F, van der Dijs B, Orozco B, et al. Plasma neurotransmitters, blood pressure and heart rate during supine-resting, orthostasis and moderate exercise conditions in major depressed patients. Biol Psychiat 1995; 38: 166-73.

5. Gobbi G, Mirandola P, Tazzari PL, et al. Flow cytometry detection of serotonin content and release in resting and activated platelets. Br J Haematol 2003; 121 $892-6$

6. Dale GL, Friese P, Batar P, et al. Stimulated platelets use serotonin to enhance their retention of procoagulant proteins on the cell surface. Nature 2002 415: 175-9.

7. Dominguez V, Govezensky T, Gevorkian G, et al Low platelet counts do not cause bleeding in an experimental model of immune thrombocytopenic purpura in mice. Haematologica 2003; 88: 679-87.
8. Lechin F, van der Dijs B. Platelet serotonin and thrombostasis. J Clin Invest 2004; (0http://www.jci.org - Letters) April 21.

9. Lechin F, van der Dijs B. Platelet activation and catheter intracoronary brachytherapy. Heart online 2004; (http://heart.bmjjournals.com Read eLetters) July 1.

10. Lechin F, van der Dijs B, Orozco B, et al. Elective stenting, platelet serotonin and thrombotic events. Platelets 2004; $15: 462$.

11. Lechin F, van der Dijs B. Platelet aggregation, platelet serotonin and pancreatitis. J Pancreas 2004; 5 : 237-9. 